\title{
A remark on quantum cloning
}

\author{
Christian de Ronde ${ }^{* 1,2,4}$, Massimiliano Sassoli de Bianchi ${ }^{1,20}$ \\ ${ }^{1}$ Vrije Universiteit Brussel, Center Leo Apostel for Interdisciplinary Studies, Brussels, Belgium \\ ${ }^{2}$ CONICET - Universidad de Buenos Aires, Instituto de Filosofía Dr. A. Korn, Buenos Aires, Argentina \\ ${ }^{3}$ Laboratorio di Autoricerca di Base, Lugano, Switzerland \\ ${ }^{4}$ Universidad Nacional Arturo Jauretche, Instituto de Ingeniería, Buenos Aires, Argentina
}

Received on February 12, 2019; Revised on March 5, 2019; Accepted on March 6, 2019.

\begin{abstract}
Physical entities are not truly teleported in so-called quantum teleportation, only their states are. Similarly, physical entities are not truly cloned in so-called quantum cloning, only their states are. The celebrated no-cloning theorem tells us that a universal cloning machine, able to perfectly clone arbitrary states, cannot be built. However, this only applies to discernible quantum entities. For the indiscernible ones, which are already perfect clones of one another, the additional cloning of their state is in fact a natural process, which happens in a spontaneous way, as soon as they are allowed to interact.
\end{abstract}

Keywords: Quantum cloning; No-cloning theorem; Indiscernible entities

A clone is a 'perfect copy of something', and to clone something means 'to produce a perfect copy of it'. Imagine taking a picture of a famous oil painting, say the Mona Lisa, using a camera of the highest quality, then printing it on paper, also using a printer of the highest quality. Would this result in a perfect copy of the original artwork by Leonardo da Vinci? Obviously not, and for a number of reasons, an important one being that the paper and inks used to print the picture, that is, to carry the patterns of the painting (its shapes and shades of color) are different from the wood panel and oils used to create the original piece. Would you want to create a perfect copy of the painting - namely, a perfect clone - you would then need not only to recreate the pictorial patterns in all possible details, but also use exactly the same substances that were used to make these patterns manifest in the first place. Indeed, a clone of a painting, to be such, should be impossible to differentiate from the original.

In our Mona Lisa metaphor, the entity in question can be considered to be the wood panel and the oils used by the artist, whereas the different possible states of the entity would correspond to the pictorial patterns that can in principle be created with these materials. To make the example a little more interesting, you can imagine that the colors never completely dry up, so that the artist can always intervene to easily modify the pictorial pattern, say transforming the "Mona Lisa smile" into a "Mona Lisa grunt." So, the wood panel and oils would always remain the same, whereas the state of the system formed by these substances can change with time.

In physics, we also distinguish an entity from its states. Generally speaking, a physical entity can be considered

*Endereço de correspondência: cderonde@gmail.com to be a collection of properties that are clustered together, where a property can be generally understood as a state of prediction towards a certain experiment, precisely testing the property in question [1]. Some of the entity's properties will be intrinsic, in the sense that they always remain actual for as long as the entity exists. These are the properties used to characterize the very identity of the entity in question. For example, the rest mass, the electric charge and the one-half fractionality of the spin of an electron are intrinsic properties distinguishing an electron from other micro-entities, like protons, neutrons, etc.

Now, if we would be attempting to create a perfect clone of a microscopic (quantum) entity, the same remark applies: in order to clone the state of the entity, one should be able to use an identical "carrier." This means that if we want to make a perfect copy of an electron in a given state, we must use another electron; if we want to obtain a perfect clone of a photon in a given state, we must use another photon, and so on.

But although an electron's spin will always remain a one-half spin, its state, that is, the 2-dimensional complex vector representing it in a 2-dimensional Hilbert space (or, equivalently, the 3 -dimensional real vector representing it in a 3-dimensional Bloch sphere) will typically change with time, and non-intrinsic properties can be associated with it, like the property of the spin to be oriented upwards with respect to a given spatial axis. Non-intrinsic properties, different from the intrinsic ones, can be either actual or potential, depending on whether the outcome of the experimental test defining the property can be predicted with probability equal to one or different from one, respectively (see for instance [2], for a discussion 
about the difference between intrinsic and dynamical properties).

That being said, we can observe that the famous 'quantum no-cloning theorem' is a statement only about the non-intrinsic properties of an entity. More precisely, the theorem says that it is impossible to make a perfect copy of an arbitrary state of a quantum entity [3 5]. The proof of the theorem is extremely simple and consists in assuming that the action of a cloning machine can be ideally described by a unitary operator $U$, such that:

$$
U\left|\psi_{i}\right\rangle \otimes\left|\psi_{0}\right\rangle=\left|\psi_{i}\right\rangle \otimes\left|\psi_{i}\right\rangle
$$

for all $i=1,2, \ldots$, where $\left|\psi_{0}\right\rangle$ is an initial "ready" state and the $\left|\psi_{i}\right\rangle$ are the states to be copied. But if we assume that, say, $\left|\psi_{1}\right\rangle$ and $\left|\psi_{2}\right\rangle$ are two orthogonal states, then for a superposition state $\left|\psi_{3}\right\rangle=a_{1}\left|\psi_{1}\right\rangle+a_{2}\left|\psi_{2}\right\rangle$, with $\left|a_{1}\right|^{2}+\left|a_{2}\right|^{2}=1$, we must also have $U\left|\psi_{3}\right\rangle \otimes\left|\psi_{0}\right\rangle=$ $\left|\psi_{3}\right\rangle \otimes\left|\psi_{3}\right\rangle$. However, by linearity:

$$
\begin{aligned}
U\left|\psi_{3}\right\rangle \otimes\left|\psi_{0}\right\rangle & =a_{1} U\left|\psi_{1}\right\rangle \otimes\left|\psi_{0}\right\rangle+a_{2} U\left|\psi_{2}\right\rangle \otimes\left|\psi_{0}\right\rangle \\
& =a_{1}\left|\psi_{1}\right\rangle \otimes\left|\psi_{1}\right\rangle+a_{2}\left|\psi_{2}\right\rangle \otimes\left|\psi_{2}\right\rangle,
\end{aligned}
$$

which is clearly different from $\left|\psi_{3}\right\rangle \otimes\left|\psi_{3}\right\rangle$, unless either $a_{1}=0$, or $a_{2}=0$.

In other words, the no-cloning theorem is a statement about the impossibility of finding a transformation that would be able to copy an arbitrary state, without altering the original, with the assumption that the state of the composite system formed by the entity whose state must be copied and the entity which has to "carry" the copied state, can be written as a tensorial product $\left|\psi_{i}\right\rangle \otimes\left|\psi_{0}\right\rangle$. This is a strong assumption, implying that the two entities in question are not entities of the same kind, i.e., entities sharing the same intrinsic properties [6]. For instance, they are not two electrons, or two photons. Or, if they are of the same kind, say two electrons, then to justify that their overall state can be described as a tensor product, one needs to avoid the two electrons to directly interact, for instance keeping them isolated inside two spatially separated boxes, so as to ensure that there are properties remaining stably actual that allow at any moment to distinguish them. However, if precautions of the above kind are not taken, this will not in general be the case.

More precisely, assume that the entity to be cloned is an electron (respectively, a photon) in state $\left|\psi_{1}\right\rangle$, and that for the cloning we use another electron (respectively, photon), in the initial state $\left|\psi_{0}\right\rangle$. We know from the 'spin-statistics theorem' that identical half-integer (respectively, integer) spin entities must satisfy the FermiDirac (respectively, Bose-Einstein) statistics, hence their state must be antisymmetric (respectively, symmetric) with respect to an exchange of the individual entities [7]. This means that in this situation a cloning machine would have no way to distinguish the state to be copied from the "ready" state to be transformed into that state. Also, in the case of fermions, Pauli's exclusion principle would forbid to have more than one entity in the same pure quantum state, thus excluding a priori the possibility of a perfect cloning of fermionic entities having the same intrinsic properties.

The above seems to go in the same direction of the no-cloning theorem, but, is it really so? Here indeed one has to face the known difficulty of ascribing individual states to entities forming a composite system when in an entangled state, as it will be typically the case when in the presence of identical entities described by symmetric or antisymmetric states. In fact, for a bipartite system formed by two identical fermions/bosons, the antisymmetric/symmetric vector state can be generally written as:

$$
\left|\Psi_{\mp}\right\rangle=c(|\psi\rangle \otimes|\phi\rangle \mp|\phi\rangle \otimes|\psi\rangle),
$$

where $c=\left(2+2|\langle\phi \mid \psi\rangle|^{2}\right)^{-\frac{1}{2}}$ is the (symmetrical) normalization factor.

When the fermionic/bosonic bipartite system is in state $\left|\Psi_{\mp}\right\rangle$, one cannot attach individual vector-states to its two components. This is another way to state what we already said above, that there is no way a cloning machine can distinguish which state is the state to be copied and which state is the state to be transformed into the state to be copied, precisely because we are in the presence of truly indiscernible entities. So, do we really need a cloning machine in this situation?

Note that the standard quantum formalism, when asserting that genuine states are only described by unit vectors (up to an overall phase factor), confronts us with the problem of having to explain how a composite system is able to exist when its components would seem to have ceased to exist, at least if we take seriously the general principle saying that if a physical entity exists then, necessarily, it must be in a well-defined state [8,9].

A way out of this paradox is to consider that bona fide states can be described not only by unit vectors, but also by density operators, which in the case of the vectors $\left|\Psi_{\mp}\right\rangle$ correspond to the one-dimensional projection operators $\rho_{\mp}=\left|\Psi_{\mp}\right\rangle\left\langle\Psi_{\mp}\right|$. When we do so, we can deduce the individual states of the entities forming a bipartite system by simply ignoring the degrees of freedom of the other component of the system (and consequently, also the way the two parts are possibly connected), which can be done by taking the partial traces of $\rho_{\mp}$.

As noted a long time ago by [10], the result of this procedure, of partially tracing out degrees of freedom, is not something that can be interpreted as a classical mixture of states, in accordance with an 'ignorance interpretation', which is the reason why he called these traced-out states 'improper mixtures', which many physicists consider today to be states in their own right. This also follows from the recent 'extended Bloch representation' of quantum mechanics, in which density operator-states also play a role in deriving the Born rule, explaining the quantum measurement processes and quantum entanglement 11 .

Now, if we calculate the two partial traces $\operatorname{Tr}_{2} \rho_{\mp}$ and $\operatorname{Tr}_{1} \rho_{\mp}$, over the second and first sub-system, which 
would correspond to the states of the first and second sub-system, respectively, after a simple calculation one finds that $\operatorname{Tr}_{2} \rho_{\mp}=\operatorname{Tr}_{1} \rho_{\mp}$, i.e., that the two sub-systems are exactly in the same (operator) state. In other words, if we agree that identical systems remain in well-defined individual states, which are described by traced-out density operators, then there is no need for cloning machines, if we are interested in a full-fledged cloning process, as quantum identical entities, be them fermions or bosons, as soon as they are allowed to interact, they all end up in exactly the same state, i.e., they all end up being the perfect clone of each other, in a spontaneous way.

To conclude, we have emphasized in this note that the celebrated no-cloning theorem of quantum mechanics only refers to discernible entities, or to entities that, if they have the same intrinsic properties, are then kept in conditions such that they are not allowed to directly interact, so as not to be subjected to the spin-statistics theorem. This is what is implicitely assumed in most descriptions of cloning machines, when for instance the state of an ion in a trap in Alice's laboratory is to be transferred into an ion contained in an identically constructed trap in Bob's laboratory, where what is meant by "the state of the system" is just the internal state of the ion, and not also that component of the state describing its centre of mass.

We have also emphasized that when considering quantum entities that have the same intrinsic properties, which is what one would expected to use for obtaining full-fledged physical clones, then there is no no-cloning theorem, as indiscernible entities all naturally acquire the same operator-state (i.e., they become not only indiscernible as regards their intrinsic properties, but also as regards their non-intrinsic ones).

Of course, in no way the present note wants to diminish the interest of quantum cloning as usually understood, i.e., cloning only referring to the copy of an entity's state. Indeed, it is precisely this possibility of copying the information about the state of an entity into the state of a different entity (within the limitations of the no-cloning theorem) having a Hilbert space of same dimension, that makes the process useful in quantum information science applications, because although the two entities are not fully identical, they are nevertheless able to produce identical statistics of outcomes in measurements.

However, we also observed that there is no discussion in the literature, or in textbooks (as far as we can judge) about the rather obvious fact that cloning, as a notion, refers primarily to a physical entity, not to its state. Quantum elementary entities being truly and strikingly indiscernible, they are always the perfect physical clones of one another, and as soon as they are allowed to interact, they become perfect copies of one another also for what concerns their individual states, at least for as long as one accepts to complete standard quantum mechanics by also including operator-states as a description of genuine individual states 12 .
In other words, the quantum no-cloning theorem is, in a sense, at odds with the all-cloning aspect that is built in quantum mechanics, resulting from quantum indistinguishability and the spin statistics theorem. When taking seriously the notion of cloning, instead of a 'nocloning theorem' we have a 'no-no-cloning theorem': when identical entities are brought together, they must be in the exact same operator-state, as indistinguishability forces entanglement and in turn entanglement (here as a symmetrical or anti-symmetrical constraint) forces the individual entities to acquire the same density matrix state.

\section{References}

[1] D. Aerts, in: Interpreting Bodies: Classical and Quantum Objects in Modern Physics, edited by E. Castellani (Princeton University Press, Princeton, 1988), p. 223.

[2] C. Ronde and C. Massri, Cadernos da Filosofia da Ciencia 2, 107 (2017).

[3] D. Dieks, Phys. Lett. A. 92, 271 (1982).

[4] W. Wootters and W. Zurek, Nature 299, 802 (1982).

[5] J. Ortigoso, Am. J. Phys. 86, 201 (2018).

[6] S. French and D. Krause, Identity in Physics: A Historical, Philosophical, and Formal Analysis (Oxford University Press, Oxford, 2006).

[7] I. Duck and E.C.G. Sudarshan, Am. J. Phys. 66, 284 (1998).

[8] B.C. van Fraassen, Quantum Mechanics: An Empiricist View (Oxford University Press, Oxford, 1991).

[9] D. Aerts, Int. J. Theor. Phys. 39, 485 (2000).

[10] B. d'Espagnat, Conceptual Foundations of Quantum Mechanics (Addison-Wesley, Reading Mass., 1976).

[11] D. Aerts and M. Sassoli de Bianchi, J. Math. Phys. 57, 122110 (2016).

[12] D. Aerts, M. Sassoli de Bianchi and S. Sozzo, Int. J. Theor. Phys. 56, 3727 (2017). 